\title{
Study of electrochemical discharge machining technology for slicing non-conductive brittle materials
}

\author{
W.Y. Peng*, Y.S. Liao \\ Department of Mechanical Engineering, National Taiwan University, No. 1, Sec. 4, Roosevelt Road, Taipei 106, Taiwan, ROC
}

Accepted 15 November 2003

\begin{abstract}
The electrochemical discharge machining (ECDM) has been proved to be a potential process for the machining of high-strength non-conductive materials. Traveling wire electrochemical discharge machining (TW-ECDM), a newly developed technology, is used to slice the small size (10-30 mm diameter) optical glass and quartz bars. The electrical-thermal etching effect and its feasibility are investigated. The energy release intensities and their physical phenomena under different sizes of discharge wires, power source modulations and methods of electrolyte supply are discussed. The pulsed dc power proves better spark stability and more spark energy release proportion than constant dc power. The input power is modulated to obtain the appropriate frequencies and duty factors for machining glass and quartz materials. The ion translation rate, the electrolyte immersing depth and the concentration of the alkali are found to be the dominant factors of bubbles reaction. Based on the SEM photographs of the workpiece surface, it is noted that the more purple the sparks from the mixed gases of hydrogen and vapor, the better the etching effect is. The V-shape defect occurring at the cut-in and cut-out can be significantly reduced by rotating the workpiece. Finally the appropriate cutting conditions for the quartz and borosilicate optical glass materials are concluded.

(C) 2004 Elsevier B.V. All rights reserved.
\end{abstract}

Keywords: Eelectrochemical discharge machining; Quartz machining; Pulsed dc power; Spark stability; Optical glass

\section{Introduction}

The EDM technology has been successful in many machining applications. It is not a brand new idea to machine non-conductive material by electrical discharge machining. But there are only few tests to limited types of materials under quite restricted conditions. The traditional method for slicing these brittle materials, such as quartz or ceramics, is mainly dependent on the direct or non-direct grinding force of hard particles. Some non-traditional procedures, like the laser machining or ultrasonic machining may be integrated to become a composite machining procedure. So far, it is still necessary to provide more study for machining of non-conductive brittle materials since they have become key materials in the MEMS field. For example, the glass or quartz is usually bonded with the semi-conductive material due to their transparency, chemical-resistant properties and so on. Likewise, the engineering ceramics is also used more and more often in the high-tech apparatus. But it becomes more difficult to be further machined once they are highly

\footnotetext{
* Corresponding author. Tel.: +886-2-23660443; fax: +886-2-23660443.

E-mail address: d88522006@ntu.edu.tw (W.Y. Peng).
}

sintered. The machining by means of grinding results in many micro-cracks owing to the direct-contact force. The crack propagation causes difficulty in realizing the fine structures on a ceramic base. A newly but not well-developed method called ECDM in recent years is introduced for this purpose. The emphasis is on the slicing process in this paper. Some investigations about ECDM have been conducted for a period of time. But the application is still limited. Some results of ECDM [1-4] are called spark assisted chemical etching (SACE) facilitate the study of TW-ECDM. The theoretical analysis is divided into the bubble generation process and the arc discharge process. As to the machining application, the discharge effects are more emphasized. The thermal etching rate had been simulated with FEM tool by Basak and Ghosh [5], and the bubble phenomena was recently analyzed by Wuthrich [6] to predict the critical voltage and the machining rate of structured 3D features on the Pyrex glass. The proportion of the bubble wrapping around the cathode is estimated by a stochastic method. But the model could not be used directly in the TW-ECDM case since the scheme of the tool set-up is quite different.

Since less information is collected and discussed about TW-ECDM, and available literature on glass and quartz machining is limited, further investigation on the spark 
process effect is still needed. It has been reported by Tsuchiya et al. [7] that TW-ECDM can slice thin optical glass and engineering ceramics with a vertical line set-up. The various influences due to electrolyte type, concentrations, and power source parameters were demonstrated. However, the thickness of the workpiece cannot be larger than $1 \mathrm{~mm}$. The over-cut is serious and the cutting rate of ceramics seems not so optimistic. Nevertheless, the potential of TW-ECDM technology is illustrated. The slicing of composite material with horizontal wire set-up has been conducted by Jain et al. [8]. The material removal rate is discussed using the wire diameter of $1 \mathrm{~mm}$. The weight-loading procedure is adopted. But the precision gap control relies on a servo feed strategy if the machining quality is required. It can be a successful and innovative method since the fiber can be effectively cut by spark energy. But the size of the workpiece is also limited due to the energy intensity problem. In this study, the optical glass, quartz and $\mathrm{Al}_{2} \mathrm{O}_{3}$ engineering ceramics are to be tested for their machining effects by TW-ECDM. Some experimental results are given. The possibilities and feasibilities of using ECDM technology to slice non-conductive materials are studied from various aspects.

\section{Requirements and strategies}

Some real tests without preliminary theoretical analysis will be presented. Vividly, the spark phenomena should be first observed carefully to derive the parameters that can induce more spark energy release. The approaches to efficiently use the spark energy of ECDM in the machining requirement are studied.

\subsection{Implementation procedures}

Two experimental subjects should be included. The utmost task is to find the necessary parameters to keep the stable and intense spark ignition. Then the real operation conditions are found to cope with the different material properties. The hardware set-up includes both the mechanism module and the electronic power source module like what happens in the WEDM machine. The experiment set-up and the local spark reaction is shown in Fig. 1. The key point of the mechanism is the wire system which controls the wire speed and wire tension. The contact between the wire and the workpiece will be detected sensitively because the tiny movement of the wire could be measured by the precision optical sensor. Besides, the electrolyte supply apparatus is used to adjust the flushing conditions and maintain the temperature. The flushing design can also improve the circulation and concentration balance of the electrolyte to promote the bubble reaction. The control of the fluid level also affects the spark reaction because of the buoyancy and surface tension effects. The power source dominates the input voltage and the current release ability. The switching type dc power is compact but the peak current release tends to be restrained. Hence, the silicon circuit rectified (SCR) type dc power is adopted to maintain better voltage-current response. The pulsed power source often stimulates more instantaneous energy release so a pulse generation controller is embedded in the power source module. The frequency regulation is between $100 \mathrm{~Hz}$ and $1 \mathrm{kHz}$. The voltage is set from 45 to $90 \mathrm{~V}$. With these arrangements, several parameters can be adjusted for the mechanism and the power source module including the wire speed, the electrolyte flushing rate the voltage,
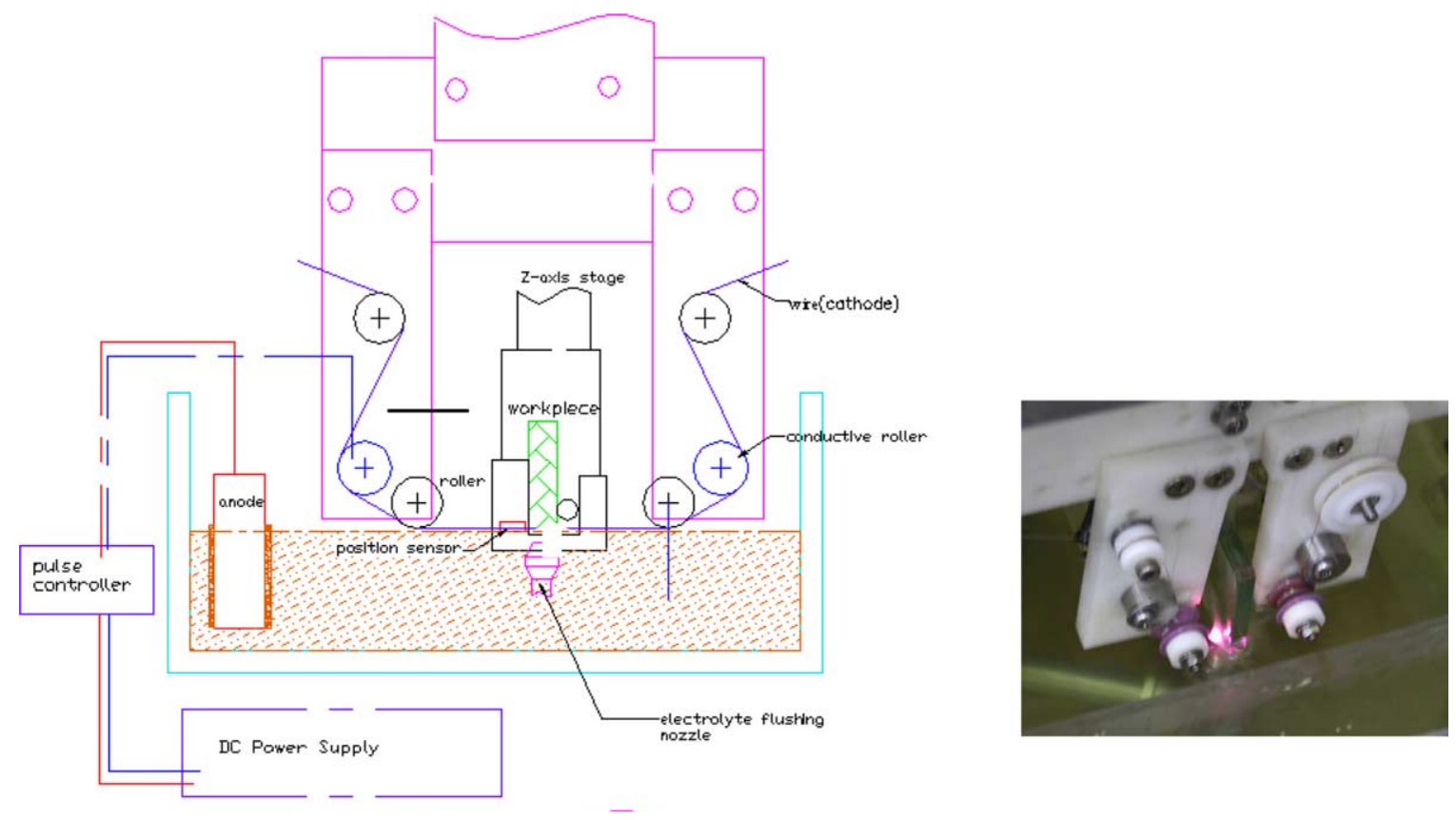

Fig. 1. The experiment set-up. 


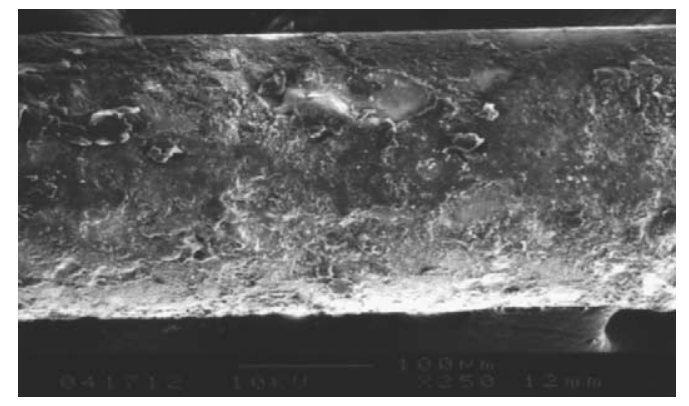

Fig. 2. The surface microstructure of the stainless wire after TW-ECDM process.

the pulse frequency, the pulse duty factor. The electrolyte will be set at $5 \mathrm{M}$ based on some existing data of ECDM process.

\subsection{Experimental methodology and preliminary observations}

The currently available copper WEDM wire and the tempered stainless wire are tested to testify if each of them fits the TW-ECDM application. It is observed that the former can evoke more sparks which result from the lower surface contact resistance and it has stable and ideal performance if the wire is no larger than $40 \mathrm{~mm}$. However, the stainless wire can sustains higher voltage and stronger erosive destruction. The little voltage drop due to the larger internal resistance of stainless material is acceptable in the pulsed circuit. The $0.25 \mathrm{~mm}$ diameter wire is used and the wire reactive length ranges from 10 to $70 \mathrm{~mm}$. The thermal etching surface on the wire is shown in Fig. 2. More craters are found on the side close to the workpiece. The wire tension is kept under $8 \mathrm{~N}$ which is not too large for $0.25 \mathrm{~mm}$ stainless wire, but the concentrated thermal input can result in wire breakage.

The spark intensity is evaluated by two schemes. The first approach is to observe directly the spark colors and distributions along the wire which reflect the temperature of the mixed vapor and the wrapping proportion of the evoked sparks. The more purple or blue the spark color, the stronger the spark is. This occurs within only certain range of conditions, and the wire breakage tends to occur if the duty factor is larger than 0.4 at this moment. The frequency variation of the spark sound can also reflect the spark intensity. Alternatively, the oscilloscope is used to check the pulse current quantitatively and if the corresponding parameters are appropriate for practical slicing machining. The average released power is measured by the product of the average current and the voltage. This corresponds to the sum of the energy of electrolysis reaction and the spark process. The more the later part is favored but these two parts of energy have their own balance regulations. Some adjustments can produce a little more spark energy which tends to be beneficial for machining. These adjustments of power source parameters, material properties and electrolysis characteristics are investigated.

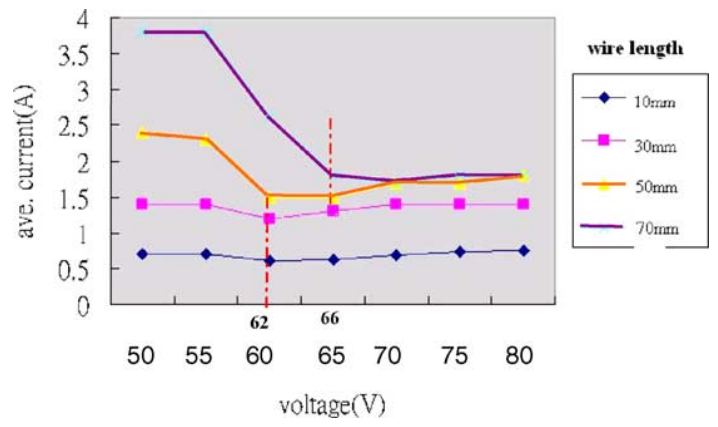

Fig. 3. The transition voltages of different wire lengths.

\section{Results and discussion}

\subsection{Reactive wire length}

In the process of ECDM, the critical voltage (CV) which relates to the concentration and formation of electrolyte, temperature and so on is vital for energy control. It means the spark evoking phenomena is vivid after the CV. If the voltage is a little higher than the $\mathrm{CV}$, the current and the voltage has approximate positive linear relationship. However, in the TW-ECDM, the apparent spark release with proper amount of bubble around the wire demands a voltage higher than the CV. It is defined as the transition voltage. Only when the voltage is larger than the transition voltage, the relationship between the voltage and current can be positively dependent. Four kinds of reactive wire length are implemented to discover the matching transition voltages. The average current is recorded with the voltage from 55 to $80 \mathrm{~V}$. As shown in Fig. 3, if the wire length is $10 \mathrm{~mm}$, the transition voltage is not apparent. For the concentration of $5 \mathrm{M}$ and the temperature of $40^{\circ} \mathrm{C}$, the $\mathrm{CV}$ of $\mathrm{KOH}$ is around $30 \mathrm{~V}$. This can be verified by adopting a tiny and sharp tool as the cathode in the ECDM process [6]. But the reactive surface area is quite larger for the TW-ECDM process than that of the ECDM. The longer the wire, the more vivid the transition voltage is, and the more it deviates from the critical voltage. This may be due to the difference of bubble distribution state. It is more difficult for the long wire to be wholly wrapped by the bubbles. Even if the bubble distribution over the wire surface is not $100 \%$, the sparks can proceed because there is some extent of on-off action on some segments of the wire and the voltage difference is maintained. The longer wire tends to easily break due to the concentrated arc discharge. For example, the duty factor should be kept under 0.3 if the wire length is around $70 \mathrm{~mm}$.

\subsection{Electrolyte supply}

The electrolyte experienced drastic concentration drop near the engaged wire. The ions can be replenished by natural concentration variation or be forcely supplied by the stream passing the wire. If the wire is immersed in the electrolyte, the control of the fluidic level can influence the spark 


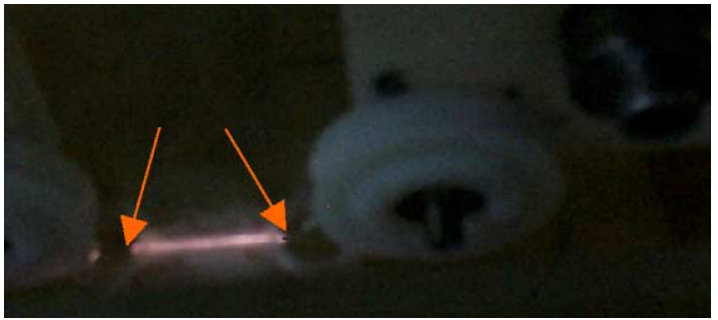

Fig. 4. The big bubble stagnating near the roller.

strength. The optimum policy is that the wire is allowed to travel on the fluidic surface and wrapped with the electrolyte film formed by surface tension. This often causes energy waste because of a segment of wire which produces sparks but does not contact the workpiece. Besides, the tiny bubble tends to combine together to form a bigger one around the roller as shown in Fig. 4.

Another way is that the electrolyte circulation could be driven by a small pump. The flowing speed and direction of the electrolyte should be coordinated with the wire speed and its traveling direction. If the electrolyte is supplied from the bottom of the workpiece, the spark light maintains purple color more easily. This results from that the electrolyte fluidic field is helpful to the hydrogen bubble reactions but the flushing speed cannot be larger than $30 \mathrm{~mm} / \mathrm{s}$ or so. The formation and wrapping process cannot be disturbed too much by the flushing effect. If the electrolyte is flushed from the top side, the flushing moment suppressing the hydrogen bubble to float up. After the wire cut-in for more than $5 \mathrm{~mm}$, the electrolyte could not circulate as well as the beginning of the machining since the narrow slot gap is not beneficial for electrolyte flowing. This can be improved a little by adjusting the flushing direction.

\subsection{Power source modulation}

It is verified that the power source type can affect the impulse current release of the ECDM process [3]. In this study, the dc power supply is connected with a pulse controller to form an adjustable power source. Because there are two sub-systems to decide the voltage-current response, the peak current mode is up to the power source characteristics and the core reactive area which is affected by the ion translation rate, the bubble size and so on.

\subsubsection{Effects of the voltage}

The voltage is positively related to each single spark intensity but it should collocate with the frequency and duty factor. Even though higher voltage can break the stability of the bubble on the wire surface and arise more severe vibration of the wire, the total spark intensity is not always proportion to the voltage. There are two categories of current pulses for the difference in their magnitude orders. The higher current pulse is about $27 \mathrm{~A}$ in Fig. 5(a) and the lower one is about $3.8 \mathrm{~A}$. The higher current impulse is aroused by the instant-on action of the pulse controller when the bubbles almost wholly wrapped the cathode. The smaller one means the insulation by bubble films is not so complete. The impulse current magnitude is important but more attention should be paid on the pulse current duration time. Because the single peak current impulse can only thermally etch one micro-spot area, frequent average current pulse release is more favored. Besides the peak current magnitude did not vary so heavily when the voltage is raised. This can be demonstrated by Fig. 5. In Fig. 5(a) and (b), the frequency is $300 \mathrm{~Hz}$ and the duty factor is 0.23 . But the voltage is $75 \mathrm{~V}$ in (a) and $85 \mathrm{~V}$ in (b). The smaller current pulse or called the current pulse of second mode is about $5 \mathrm{~A}$ in (b). This is due to the elevation of voltage. The higher current pulse or called the current pulse of first mode is almost the same. Under this frequency and reactive wire length, the voltage elevation near $70 \mathrm{~V}$ is somehow beneficial for total energy intensity. For higher voltage of above $90 \mathrm{~V}$, the wire breaks more easily but the vital current pulse of second mode is not upgraded apparently.

\subsubsection{Effects of frequency and duty factor}

The frequency and duty factor interact mutually to affect the energy intensity. Generally speaking, the frequency is positively related to the average current. But it will be excluded if the frequency is too high for the chemical reaction and certain wire length. In Fig. 6, the only difference is the voltage between (a) and (b). The frequency is $400 \mathrm{~Hz}$ in

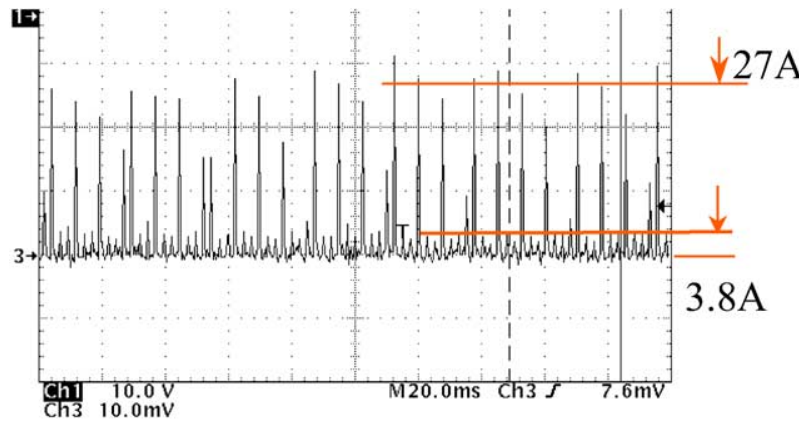

(a)

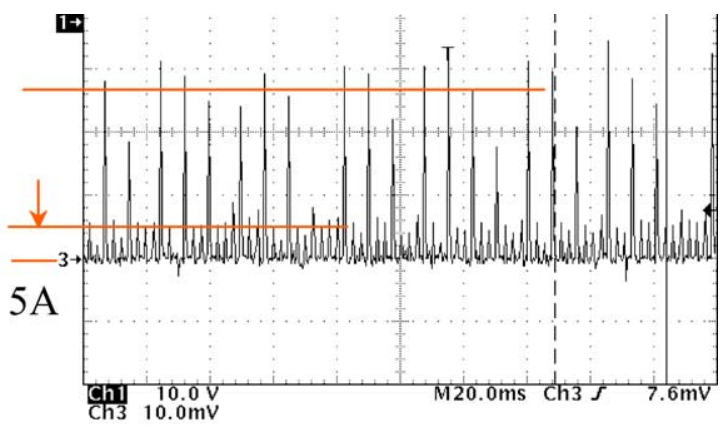

(b)

Fig. 5. The voltage and the pulse current response: (a) $75 \mathrm{~V}, 300 \mathrm{~Hz}$ and 0.23 ; (b) $85 \mathrm{~V}, 300 \mathrm{~Hz}$ and 0.23 . 


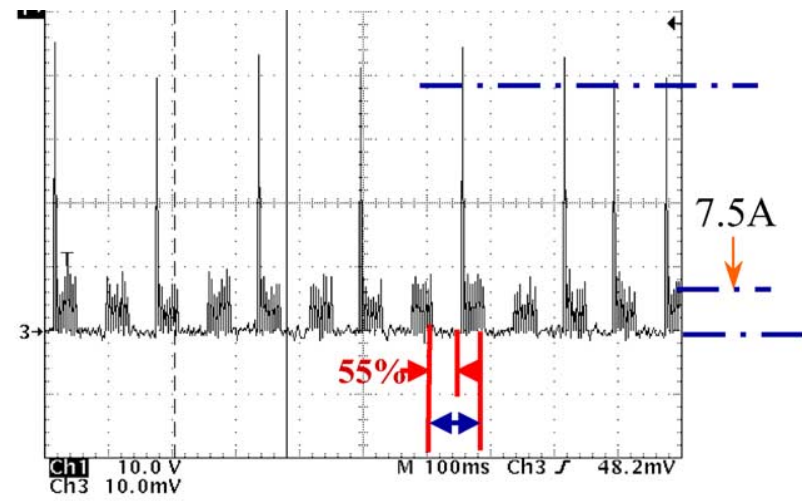

(a)

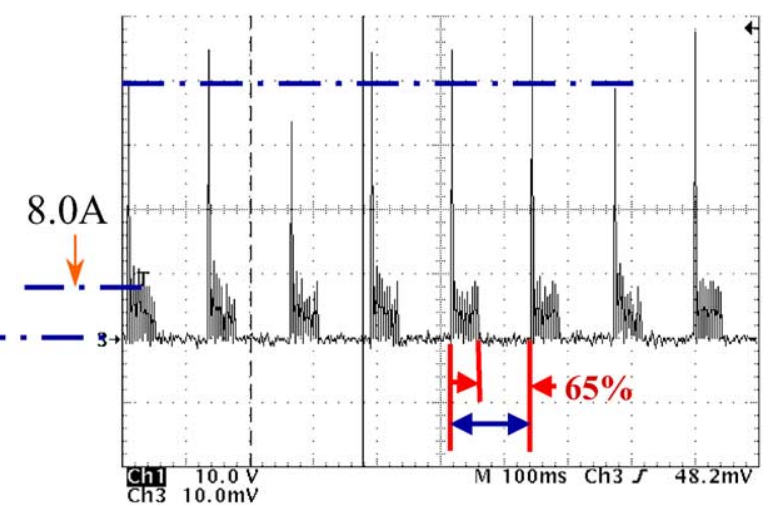

(b)

Fig. 6. The voltage and the pulse current response: (a) $75 \mathrm{~V}, 400 \mathrm{~Hz}$ and 0.23 ; (b) $85 \mathrm{~V}, 400 \mathrm{~Hz}$ and 0.23 .

Fig. 6 which is higher than that in Fig. 5. The current pulse of the second mode is little different under 75 and $85 \mathrm{~V}$. This means voltage elevation loses its effects in higher frequency for longer wire. Besides, the ECDM process is depressed among 55\% of time in average in Fig. 6(a). In the depressed period, there may be thick bubbles around the wire or it begins to produce new bubbles after a combined big bubble just leaves the wire. It shows the frequency of $400 \mathrm{~Hz}$ is too high for the wire longer than $45 \mathrm{~mm}$. The bubble circulation mechanism cannot match well with the power source frequency. If the voltage is set higher as $85 \mathrm{~V}$, it is worse since the depressed period is extended to $65 \%$ of time as shown in Fig. 6(b). This means the voltage and frequency should cooperate with the metabolic rate of the bubbles to obtain the optimum energy intensity.

As to the duty factor, if it is larger than 0.65 , the pulse current phenomena is more close to what happens in pure $\mathrm{dc}$ power source. It enables stronger and concentrated current pulse of mode one if the duty factor is between 0.2 and 0.4 and the frequency and voltage are properly matched. The ratio of the current pulse of mode one to that of mode two is about 0.13 as shown in Fig. 7. The material removal mechanism of ECDM relies on the spark energy which consists of explosive kinetic energy and large amount of heat. The duty factor should be decided after the material property is known. The melting point and mechanical strength are the most concerned material characteristics for the real TW-ECDM process. If the melting point and mechanical strength of the material is as those of the optical glass, a higher duty factor from 0.4 to 0.55 which promotes higher ratio of the pulse current of mode two can cover the thermal etching demand well. However, a lower duty factor of about 0.25 is more beneficial for slicing ceramic materials because there will be more pulse current of mode one to etch the surface structure of the high-strength material.

\subsection{Material tests}

\subsubsection{Optical glass and quartz}

For the optical glass such as pyrex, the melting point is around $1000{ }^{\circ} \mathrm{C}$, and that of the quartz is about $1700^{\circ} \mathrm{C}$. The gap control plays an important role because most of the arc discharge intensity ranges between 1 and $5 \mathrm{~A}$ in ECDM. The voltage cannot be too large to stimulate severe wire vibration which destroys the stable gap. In the test, the thickness of the borosilicate glass and quartz is $10 \mathrm{~mm}$. The $\mathrm{KOH}$ electrolyte has stronger spark effects but the chemical etching is more severe comparing to $\mathrm{NaOH}$. The two SEM photos in Fig. 8 shows the cut-in slot and the microstructure on the side surface with the $\mathrm{KOH}$ electrolyte. Because of the current pulse of mode one and the thermal stress effect, the micro-cracks initiate. This can be reduced by adjusting the voltage and

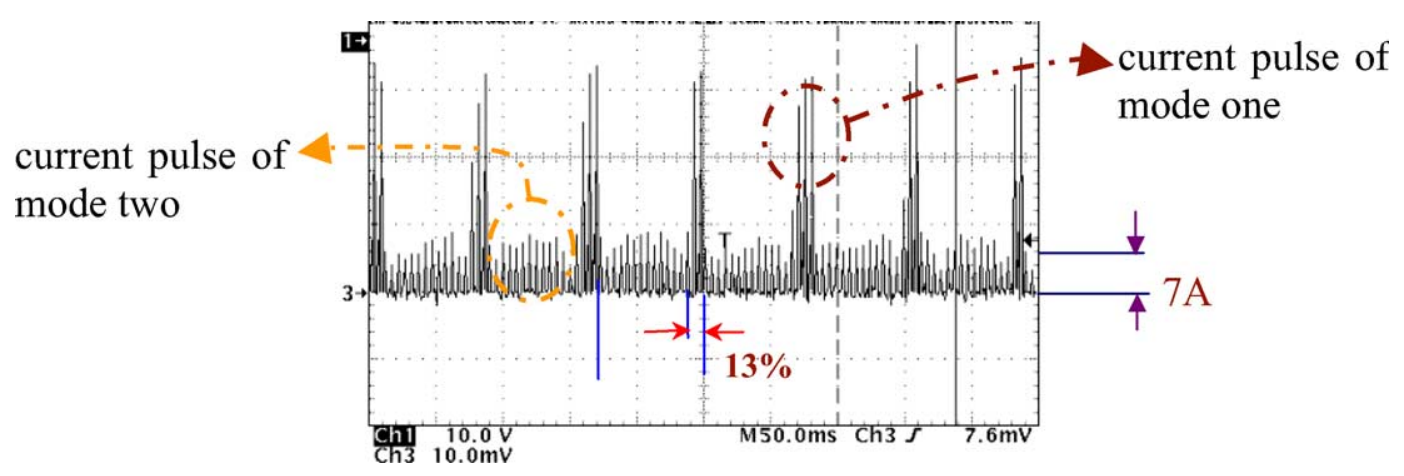

Fig. 7. The ratio of the current pulse of mode one and two. 

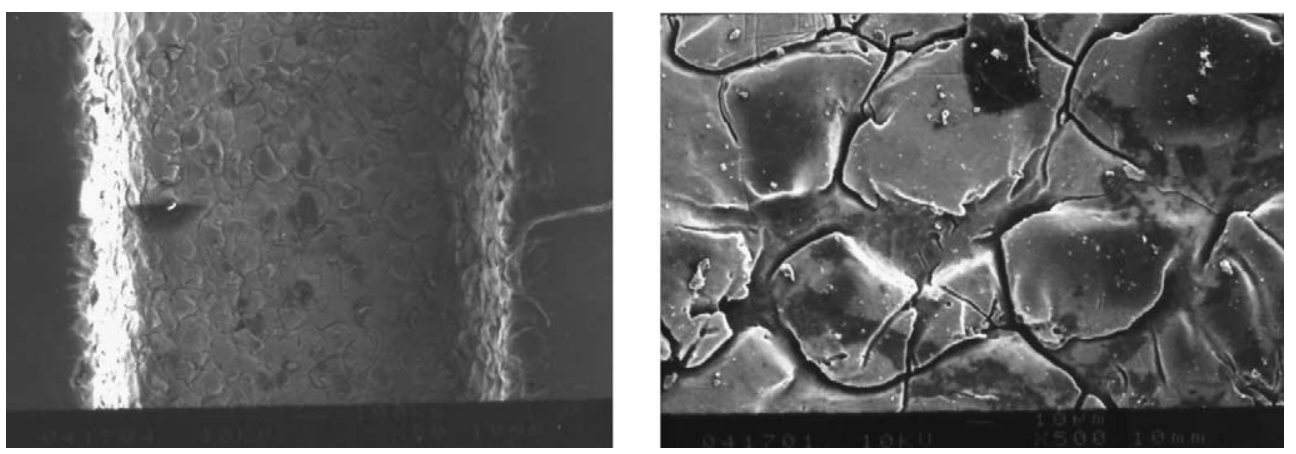

Fig. 8. The cutting slot and microstructure of the borosilicate glass.

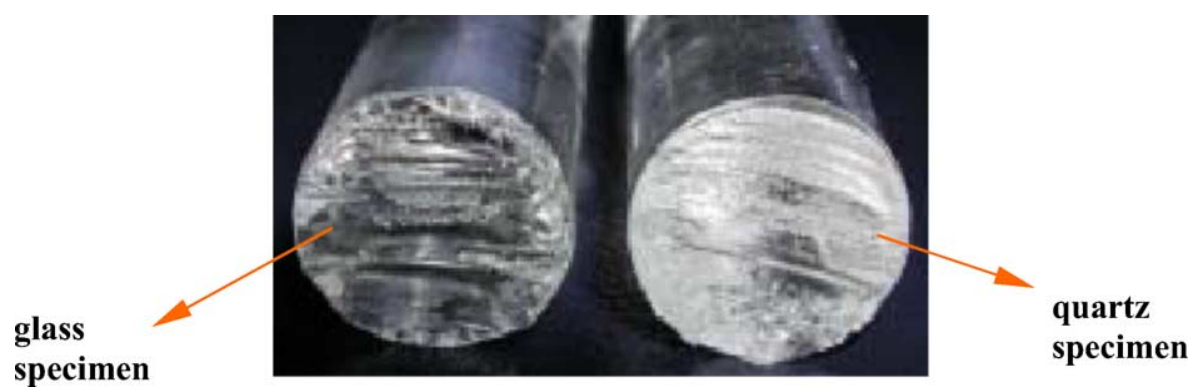

Fig. 9. The cross-sectional views of the sliced glass and quartz.

frequency and using the $\mathrm{NaOH}$ electrolyte. It is found that the material removal rate (MRR) of $0.9 \mathrm{~mm}^{3} / \mathrm{min}$ can be obtained using the duty factor of 0.53 , the frequency of $200 \mathrm{~Hz}$ and the voltage of $65 \mathrm{~V}$. As to the quartz material, there are fewer surface micro-cracks if it is experienced with more pulse current of first mode due to its stronger mechanical properties. The cross-sectional views of the sliced glass and quartz bars are shown in Fig. 9. The end face of the sliced glass shows better transparency and surface roughness because it is more easily etched by the alkali. The voltage for

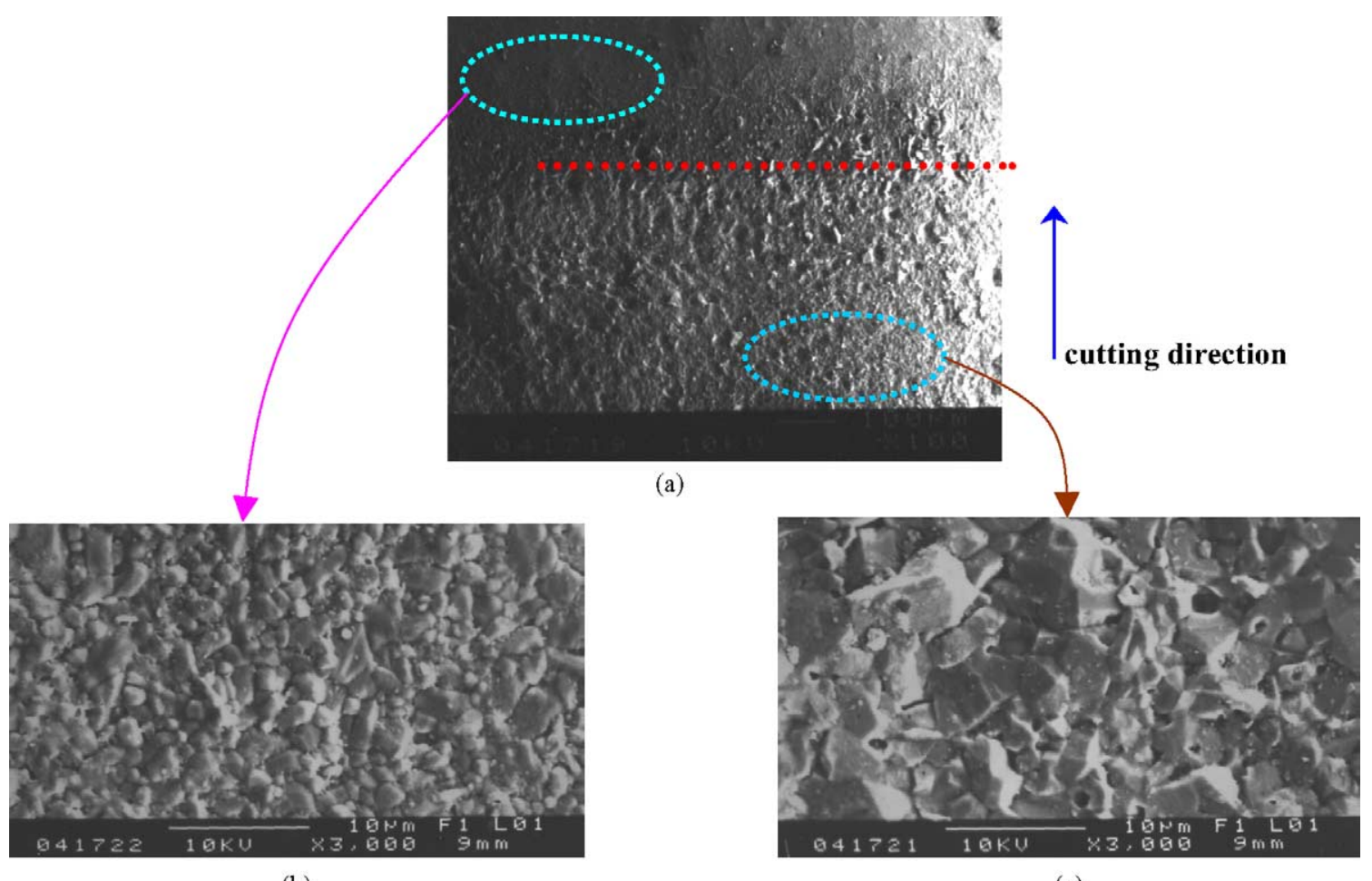

(b)

(c)

Fig. 10. The microstructure of the $\mathrm{Al}_{2} \mathrm{O}_{3}$ ceramics before and after TW-ECDM testing. 
slicing quartz should be higher, around $75 \mathrm{~V}$ and the duty factor be lower, around 0.3 to keep a MRR of $0.6 \mathrm{~mm}^{3} / \mathrm{min}$. Besides, the frequency around $300 \mathrm{~Hz}$ is more favored since it enables more current pulse of mode one. It is suggested that the rotation of a bar specimen can improve the quality of the sliced surface since the reactive wire length could be reduced and the energy control is easier.

\subsubsection{Engineering ceramics}

Apparently there needs higher energy intensity to slice the engineering ceramic material since it inherits higher strength and melting point. The $\mathrm{Al}_{2} \mathrm{O}_{3}$ engineering ceramic plate is tested under higher voltage around $85 \mathrm{~V}$. The frequency is $300 \mathrm{~Hz}$ and the duty factor is 0.23 . The $\mathrm{KOH}$ electrolyte is $6 \mathrm{M}$ and the temperature is kept at $50^{\circ} \mathrm{C}$. This set of parameters induces more current pulse of mode one which means more explosive force is applied to the ceramic surface. The MRR is about $0.06 \mathrm{~mm}^{3} / \mathrm{min}$. The surface microstructures of the specimen before and after the TW-ECDM process are shown in Fig. 10. It is vivid that the surface is more coarse after machining and the surface roughness $\left(R_{\mathrm{a}}\right)$ is about $3.5 \mu \mathrm{m}$. So far the thickness of the ceramic specimen should be no more than $2 \mathrm{~mm}$ if the wire could really penetrate the specimen by TW-ECDM.

\section{Conclusions}

It is verified that the TW-ECDM can be applied for slicing meso-size non-conductive brittle materials of several millimeters thick. The electrolyte supply is investigated for more stable and stronger spark release. The lower ambient pressure could evoke more drastic reaction. The power source is modulated to find the suitable parameters for different material properties being sliced under different energy intensity. The current pulse can be categorized as two modes. The stronger pulse current of first mode is the minority but has better machining effects for harder materials like engineering ceramics. Maybe the current pulse can be improved if voltage input is multi-step regulation and the frequency is adjusted in time according to the bubble situation.

\section{References}

[1] H. Kurafuji, K. Suda, The micro-hole making on glass by electrochemical spark machining, Ann. CIRP 16 (1968) 415.

[2] N.H. Cook, G.B. Foote, P. Jordan, B.N. Kalyani, Experimental studies in electro-machining, J. Eng. Ind. (1973) 945-950.

[3] V.K. Jain, P. Kumar, P. Rajurkar, Investigation into machining of composites, Precis. Eng. 12 (4) (1990) 227-238.

[4] I. Basak, A. Ghosh, Mechanism of material removal in electrochemical discharge machining: a theoretic model and experimental verification, J. Mater. Process. Technol. 71 (1992) 350-359.

[5] I. Basak, A. Ghosh, Mechanism of spark generation during electrochemical discharge machining: a theoretical model and experimental verification, J. Mater. Process. Technol. 58 (1996) 24-31.

[6] R. Wuthrich, V. Fascio, H. Langen, Investigations of spark assisted chemical engraving, J. Electrochem. Commun. 5 (2003) 203-207.

[7] H. Tsuchiya, T. Inoue, M. Miyazaki, Wire electro-chemical discharge machining of glass and ceramics, in: Proceedings of the Fifth International Conference on Production Engineering, Tokyo, 1984, pp. 413-417.

[8] V.K. Jain, P.M. Dixit, P.M. Pandey, On the analysis of the electro-chemical spark machining process-test of machining on composite materials, Int. J. Mach. Tools Manuf. 39 (1999) 165-186. 